\title{
Edge Detection in Digital Images Corrupted by Salt and Pepper Noise using Adaptive Neuro- Fuzzy Inference System (ANFIS)
}

\author{
Karishma Bhardwaj \\ Research Scholar \\ D.A.V.I.E.T, Jalandhar
}

\author{
Palvinder Singh Mann \\ Asst. Professor \\ D.A.V.I.E.T, Jalandhar
}

\begin{abstract}
This paper presents an Edge Detection technique for images corrupted by salt and pepper noise, which is based on an Adaptive Neuro-Fuzzy Inference System (ANFIS). This proposed technique first of all filters out the noise by Noise Adaptive Fuzzy Switching Median Filter (NAFSMF) and then find the edges using proposed ANFIS based edge detector. The training pattern for edge detection is proposed to optimize the internal parameters of the ANFIS based edge detector. The edges are directly determined by the proposed ANFIS based edge detector. This proposed edge detector is then compared with popular edge detectors Sobel, Roberts, Prewitt on the basis of performance metrics PSNR (Peak Signal to Noise Ratio), MSE (Mean Square Error) and No. of Edges detected.
\end{abstract}

\section{Keywords}

Adaptive Neuro-Fuzzy Inference System (ANFIS), Edge Detection, Salt and Pepper noise, Sobel, Roberts, Prewitt

\section{INTRODUCTION}

Edge detection is a frequently performed operation in image processing applications because it is usually the first operation that is performed before other image processing tasks such as image segmentation, boundary detection, object recognition and classification, image registration, and so on[4]. Therefore the successes of these image processing tasks are strictly dependent on the performance of edge detection.

The most important factor which decreases the performance of edge detection is the noise. Noise is an unwanted signal that degrades the visual quality of digital image. The main sources of noise in digital images are imperfect instruments, interference natural phenomena, transmission and compression [12].There is two types of noise in an image: Class Dependent Noise and Class Independent Noise. The Class Dependent noise is the noise added to each point of the image e.e.g. salt and pepper noise [11]. It has only two possible values, a high value and a low value. For an 8 bit image value of white pixel is " 1 " and for black pixel is " 0 " giving the image, Salt and Pepper like appearance. The unaffected pixels remain unchanged [12]. The Class independent noise is not dependent on the intensity of the signal (e.g. the noise in image transmission channels, the noise added to the image by the camera etc) [11].In the proposed system first of all we filter out the noise by Noise Adaptive Fuzzy Switching Median Filter (NAFSMF) and then detect Edges using Adaptive Neuro-Fuzzy Inference System( ANFIS) based edge detector.

Edge detectors may well be classified into following categories, Gradient edge detectors [13] which contains classical operators and uses first directional derivative operation. It includes algorithms such as Sobel, Roberts, Prewitt. The disadvantages of these detectors are sensitivity to noise and inaccuracy. Zero crossing [13] which uses second derivative and includes Laplacian Operator and second directional derivative. They have fixed directional characteristics in all directions but they are also very sensitive to noise. Gaussian Edge Detectors [13] includes canny operator which is symmetric along the edge and reduces the noise by smoothing the image. The advantages of canny operator are Localization and response, improving signal to noise ratio, Better detection especially in noise conditions. But they are computationally more complex than above mentioned operators.

In the system described in [5], they have proposed a novel method based on fuzzy logic reasoning for edge detection in digital images without determining the threshold value. The proposed approach begins by segmenting the images into regions using floating $3 \times 3$ binary matrixes. A direct fuzzy inference system mapped a range of values distinct from each other in the floating matrix to detect the edge by using 8 proposed rules.

In the system described in [8], they have proposed a new edge detection technique based on the BP neural network. They classified the edge patterns of binary images into 16 possible types of visual patterns. Then after training the pre-defined edge patterns, the BP neural network is applied to correspond any type of edges with its related visual pattern.

In the system described in [7], they have presented a novel adaptive neuro-fuzzy inference system (ANFIS) for edge detection in digital images. The internal parameters of the proposed ANFIS edge detector are optimized by training using very simple artificial images. The edges are directly determined by ANFIS network.

In this paper, we present an adaptive neuro-fuzzy Inference system (ANFIS) for edge detection of an image corrupted by salt and pepper noise. In the proposed method, the edges are directly determined by an ANFIS based edge detector. Then result has been compared with the standard algorithms.

\section{PROPOSED METHOD}

Fig1. Shows the Flow Chart of the proposed system. First of all we take an input image which is corrupted by salt and pepper noise. Then to filter out the noise we apply Noise Adaptive Fuzzy Switching Median Filter (NASFSMF). After that we apply ANFIS (Adaptive Neuro- Fuzzy Inference System) based Edge Detection technique to find out the edges and then compare it with Sobel edge detector, Roberts edge detector and Prewitt edge detector on the basis of performance 
metrics PSNR (Peak Signal to Noise Ratio), MSE (Mean Square Error) and No. of Edges detected and then get the final edge image

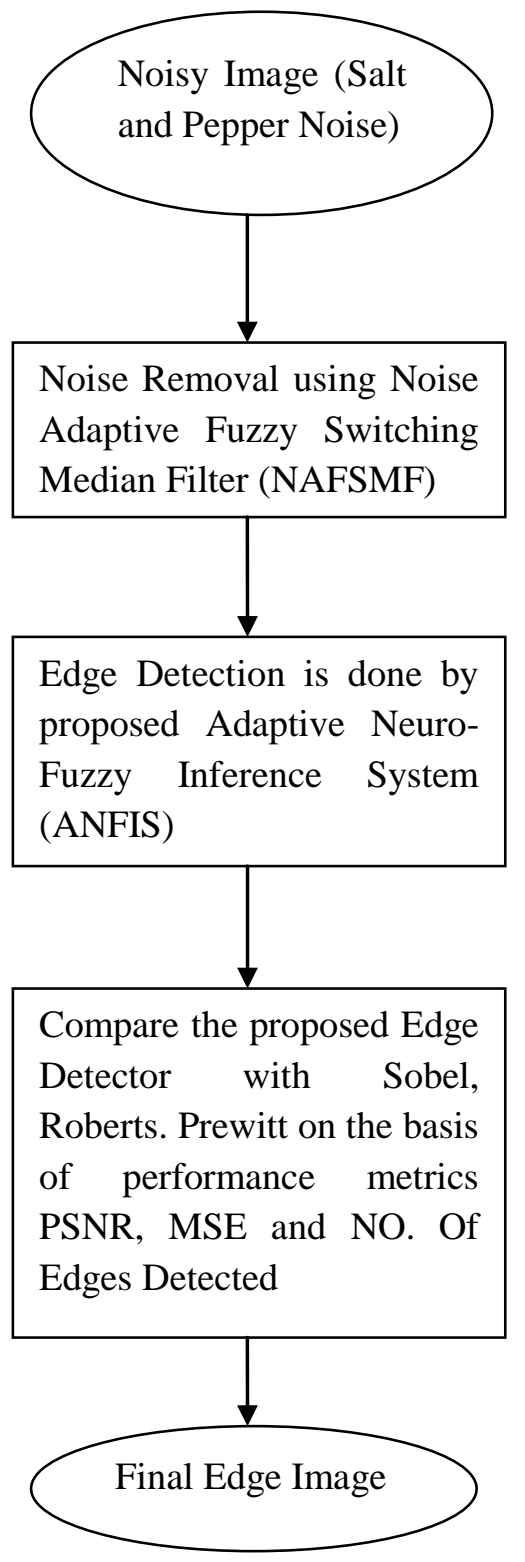

Fig 1: Flow Chart of Proposed System

\subsection{Noise Adaptive Fuzzy Switching Median Filter (NAFSMF)}

As we know the first step in Edge Detection is Filtering. Filtering means suppress as much noise as possible, without destroying the true edges. In the proposed system, firstly we convert the input image to gray scale image then we add salt and Pepper noise in the input image. The filtering of noise is done by Noise Adaptive Fuzzy Switching Median Filter (NAFSMF)[14]. It is a hybrid filter consists of simple adaptive median filter and fuzzy switching median filter. This filter removes as much noise as possible without destroying the true edges. It will speed up the filtering process at the same time preserving image detail by selecting only noise pixels for processing. Adaptive property enables the Noise adaptive fuzzy switching median filter to expand the size of its filtering window according to the local noise density. The concept of fuzzy logic is used to overcome the uncertainty in determining noisy pixel or noise free pixel.

1. First step is to detect the noisy pixels. For gray scale image, $L_{\text {salt }}=255$ and $L_{\text {pepper }}=0$. A binary noise mask $N(i, j)$ is created to find the location of "noise pixels" as follows:

$$
N(i, j)=\left\{\begin{aligned}
0, X(i, j)=L_{\text {salt }} \text { or } L_{\text {pepper }} \\
1, \text { Otherwise } \\
\ldots \ldots \ldots \ldots \ldots(1
\end{aligned}\right.
$$

Where $N(i, j)$ is binary mask and $X(i, j)$ is pixel at Location $(i, j)$ with intensity $X . N(i, j)=0$ means Noisy pixel and $N(i, j)=1$ means Noise free pixel.

2. Take $3 \times 3$ filtering window and start counting no. of noise free pixels. If no. of noise free pixels is less than minimum number of one noise free pixel then increase the window size to one pixel at each of its four sides. Then calculate the median of all noise free pixels detected. If no noise free pixel is detected we will find the mean of first four pixels in $3 \times 3$ filtering window.

3. Then we find absolute luminance difference which is the difference between center pixel and neighboring pixel.

$$
d(i+k, j+l)=|X(i+k, j+l)-X(i, j)|
$$

Then we take the maximum absolute luminance difference.

$$
D(i, j)=\max \{d(i+k, j+l) .
$$

4. Sometimes we take noise free pixel as noisy pixel. So to overcome this uncertainty some fuzzy rules are proposed.

$$
F(i, j)=\left\{\begin{array}{lll}
0 & : \quad D(i, j)<T 1 \\
\frac{D(i, j)-T 1}{T 2-T 1} & : \quad T 1<=D(i, j)<T 2 \\
1 & : \quad D(i, j)>=T 2 \ldots(4)
\end{array}\right.
$$

Where $F(i, j)$ is Fuzzy membership function. $D(i, j)$ is maximum absolute luminance difference.T1 and $\mathrm{T} 2$ are two experimentally determined parameters.

5. The detected noisy pixel is then corrected by correction term $\mathrm{Y}(\mathrm{i}, \mathrm{j})$ as follows:

$$
Y(i, j)=[1-F(i, j)] \cdot X(i, j)+F(i, j) \cdot M(i, j)
$$

Where $F(i, j)$ is Fuzzy membership function, $M(i, j)$ is the median. $X(i, j)$ is noisy pixel.

\subsection{Adaptive Neuro-Fuzzy Inference System (ANFIS) based Edge Detection}

After the noise has been removed by Noise Adaptive Fuzzy Switching Median Filter (NAFSMF), the proposed method detects the edges from the digital images by proposed ANFIS 
(Adaptive Neuro Fuzzy System) as it is a combination of Fuzzy logic and Neural Networks so ANFIS takes advantages from fuzzy logic and neural networks. This ANFIS constructs a Fuzzy inference system by using given training data set whose membership function parameters are adjusted by back propagation algorithm or in combination with least square type of method. Fig 2 shows a high level diagram of the proposed ANFIS. Inputs and their membership functions appear to the left of the ANFIS structural characteristics, while outputs and their membership functions appear on the right.

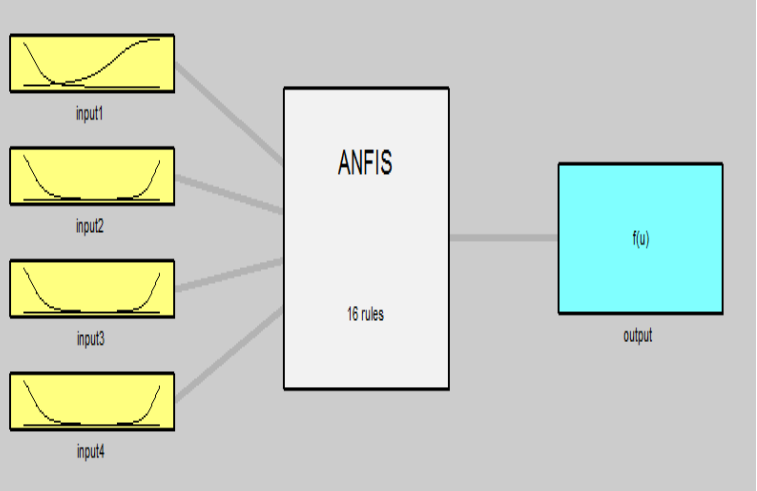

Fig 2: Diagram of Proposed ANFIS

The proposed ANFIS detector is a first-order Sugeno type fuzzy inference system with 4-inputs and 1-output. Each input has 2 generalized bell type membership functions and the output has a constant membership function as shown in Fig 3.

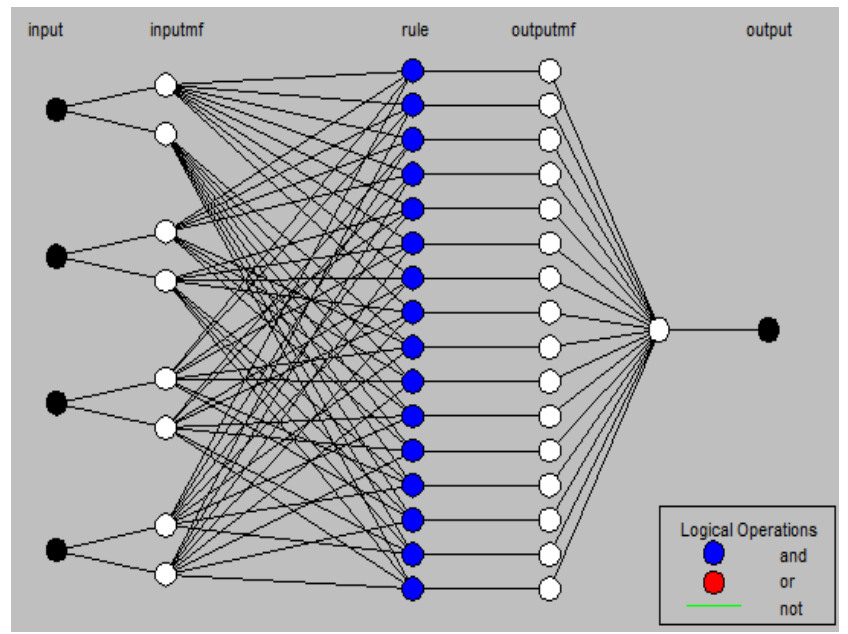

Fig 3: ANFIS Model Structure

To detect the edges we train our ANFIS with this training pattern as shown in Fig 4.This classified edge patterns in binary images into 16 categories. Then we extract set of 9 pixels by $3 \times 3$ image pixel window from the input image as shown in Fig 5 and evaluate the input image according to these 8 sub detectors as shown in Fig 6. Each sub detector has 4 inputs and 1 output. Each input has 2 generalized bell type membership functions and the output has a constant membership function and then finally we take the mean of output produced by 8 sub detectors to get final edge image.

\begin{tabular}{|c|c|}
\hline Input & Output \\
\hline 0100 & 0 \\
0101 & 1 \\
0110 & 1 \\
0111 & 1 \\
0000 & 1 \\
0001 & 1 \\
0010 & 1 \\
0011 & 1 \\
1100 & 1 \\
1101 & 1 \\
1110 & 1 \\
1111 & 1 \\
1000 & 1 \\
1001 & 1 \\
1010 & 1 \\
1011 & 0 \\
\hline
\end{tabular}

\begin{tabular}{|c|c|c|}
\hline P1 & P2 & P3 \\
\hline P4 & P5 & P6 \\
\hline P7 & P8 & P9 \\
\hline
\end{tabular}

Fig 5: Floating 3x3 pixel window mask
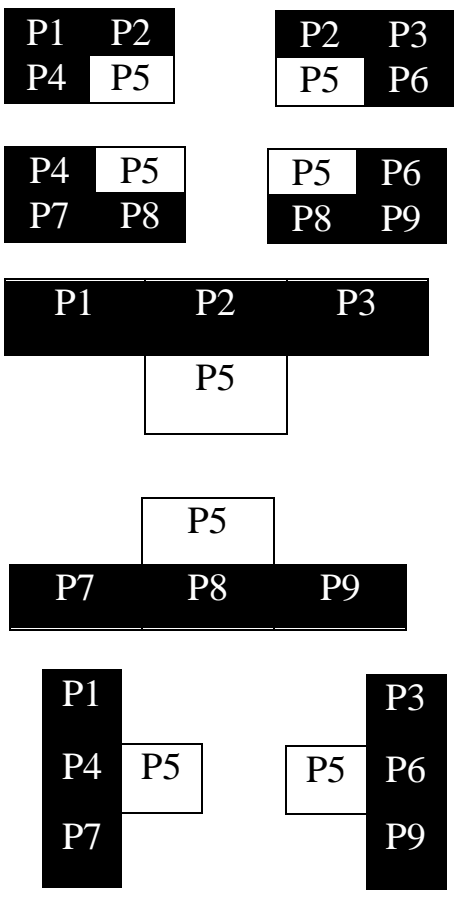

Fig 6: Eight Sub detectors 


\section{RESULTS}

The proposed edge detection method is simulated using MATLAB on different images. It is observed that this proposed system provide much more distinct marked edges as compared to Sobel, Roberts and Prewitt edge detection algorithm.

Fig 7(a),7(b),7(c),7(d),7(e) shows the Original Lena Image, Proposed Method, Roberts, Sobel and Prewitt respectively. Fig 8(a), 8(b), 8(c) shows the Graphs of MSE, PSNR and No. of Edges Detected for Original Image, Proposed Method, Sobel, Roberts and Prewitt respectively. The Table 1: shows the values of MSE, PSNR, and No. Of Edges Detected for proposed method, Sobel and Roberts edge detector using Lena Image.

Fig 9(a), 9(b), 9(c), 9(d), 9(e) shows the Original Cameraman Image, Proposed Method, Roberts, Sobel and Prewitt respectively. Fig 10(a), 10(b), 10(c) shows the Graphs of MSE, PSNR and No. of Edges Detected for Original Image, Proposed Method, Sobel, Roberts and Prewitt respectively. The Table 2: shows the values of MSE, PSNR, and No. Of Edges Detected for proposed method, Sobel and Roberts edge detector using Cameraman Image.

Fig 11(a), 11(b), 11(c), 11(d), 11(e) shows the Original Pebbles Image, Proposed Method, Roberts, Sobel and Prewitt respectively. Fig 12(a), 12(b), 12(c) shows the Graphs of MSE, PSNR and No. of Edges Detected for Original Image, Proposed Method, Sobel, Roberts and Prewitt respectively. The Table 3: shows the values of MSE, PSNR, and No. Of Edges Detected for proposed method, Sobel and Roberts edge detector using Pebbles Image.

The value of MSE is lowest for proposed edge detector and corresponding value of PSNR is highest. No. of Edges Detected by proposed method is more as compared to competing edge detectors (Roberts, Sobel and Prewitt). So it is proved that the proposed edge detector is better than competing detectors (Roberts, Sobel and Prewitt). Thus the ANFIS based System detects the edges with a very high efficiency.

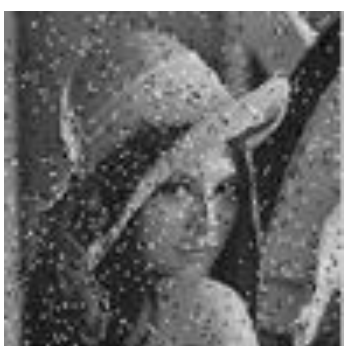

Fig.7 (a) Lena Image

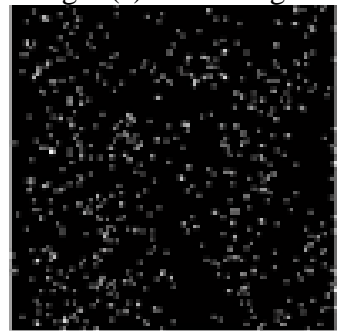

Fig. 7(c) Roberts
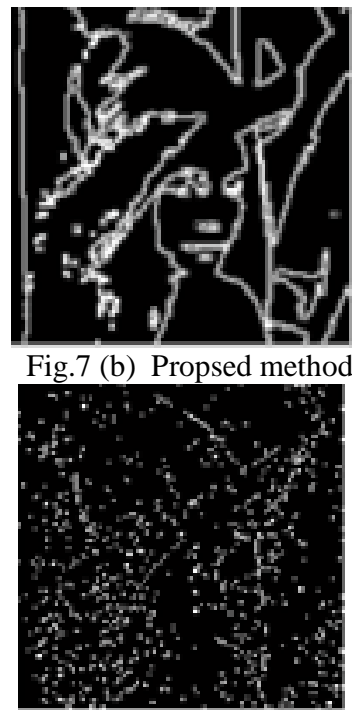

Fig. 7(d) Sobel

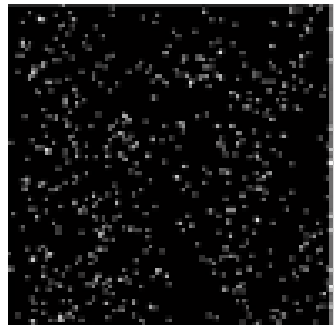

Fig. 7(e) Prewitt

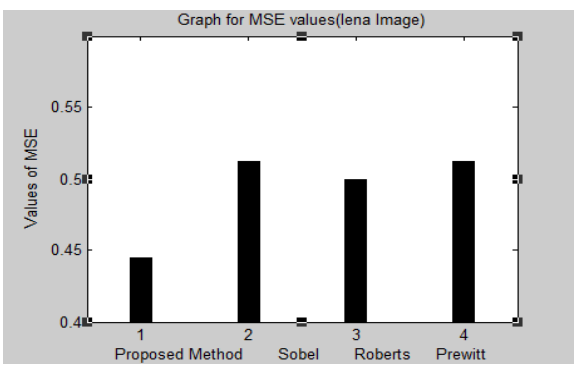

Fig. 8(a) Graph of MSE values(Lena Image)

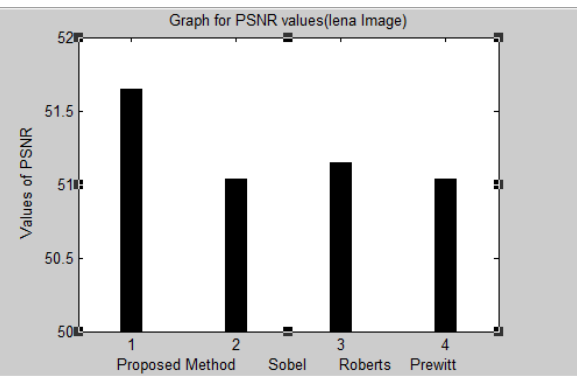

Fig. 8(b) Graph of PSNR values(Lena Image)

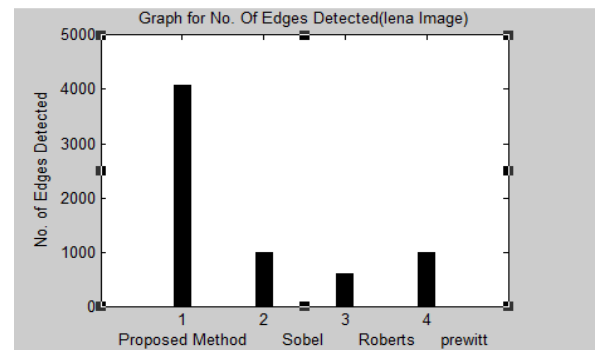

Fig 8(c)Graph of No.of Edges detected(Lena Image)

Table 1: Values of MSE, PSNR and No. of Edges Detected (Lena Image)

\begin{tabular}{|c|c|c|c|}
\hline Methods & MSE & PSNR & $\begin{array}{c}\text { No. Of } \\
\text { Edges } \\
\text { Detected }\end{array}$ \\
\hline $\begin{array}{c}\text { Proposed } \\
\text { Method }\end{array}$ & 0.4440 & 51.6568 & 4114 \\
\hline Roberts & 0.4915 & 51.2156 & 573 \\
\hline Sobel & 0.5032 & 51.2156 & 899 \\
\hline Prewitt & 0.5030 & 51.1154 & 847 \\
\hline
\end{tabular}




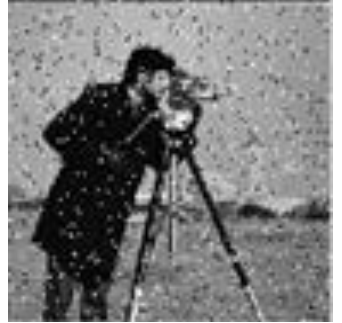

Fig. 9(a) Cameraman Image

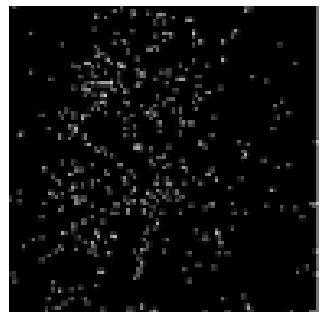

Fig. 9(c) Roberts

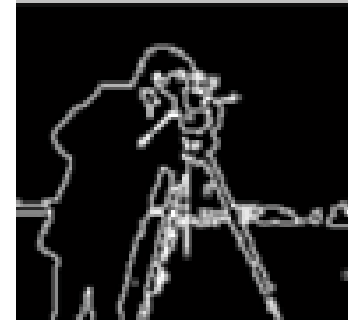

Fig. 9(b)Proposed Method

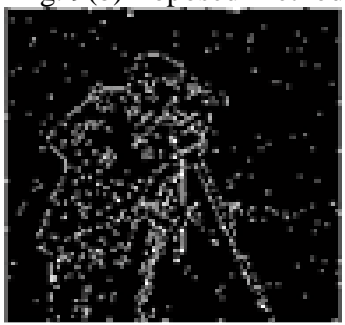

Fig. 9(d)Sobel

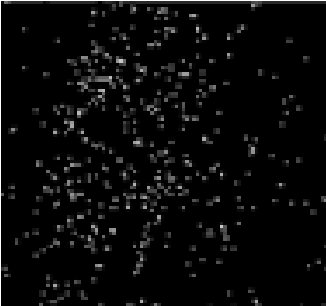

Fig. 9(e)Prewitt

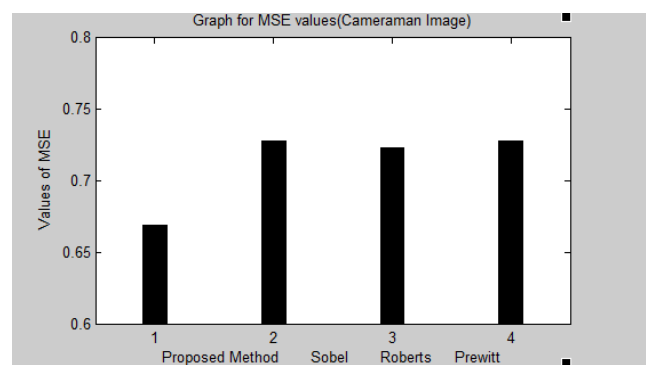

Fig. 10(a) Graph of MSE values(Cameraman Image)

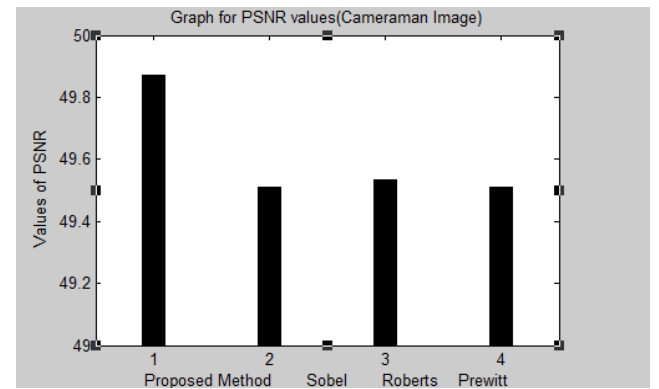

Fig. 10(b) Graph of PSNR values(Cameraman Image)

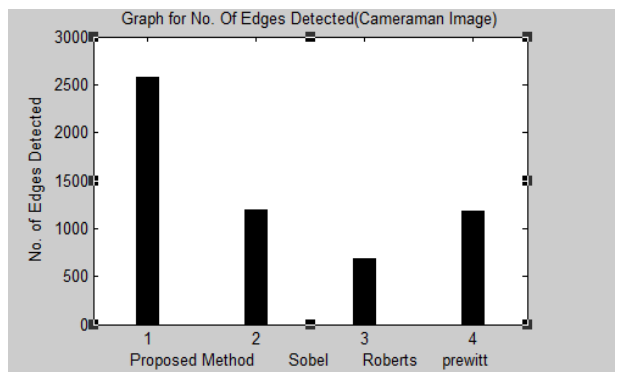

Fig. 10(c)Graph of No.of Edges detected(Cameraman Image)

Table 2: Values of MSE, PSNR and No. of Edges Detected (Cameraman Image)

\begin{tabular}{|c|c|c|c|}
\hline Methods & MSE & PSNR & $\begin{array}{c}\text { No. Of } \\
\text { Edges } \\
\text { Detected }\end{array}$ \\
\hline $\begin{array}{c}\text { Proposed } \\
\text { Method }\end{array}$ & 0.6700 & 49.8702 & 2576 \\
\hline Roberts & 0.7244 & 49.5311 & 699 \\
\hline Sobel & 0.7297 & 49.4991 & 1168 \\
\hline Prewitt & 0.7297 & 49.4995 & 1150 \\
& & & \\
\hline
\end{tabular}

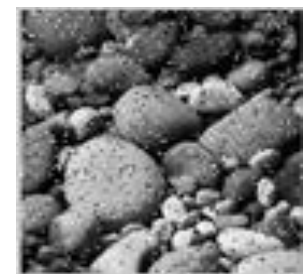

Fig. 11(a) Pebbles Image

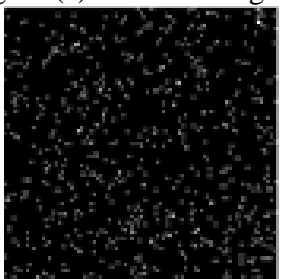

Fig. 11(c) Roberts

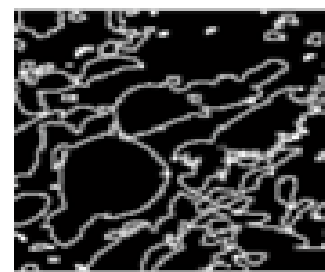

Fig. 11(b) Proposed Method

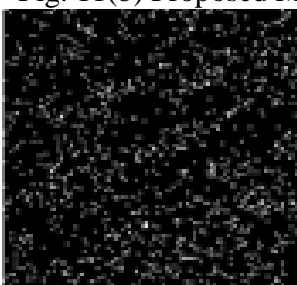

Fig. 11(d) Sobel

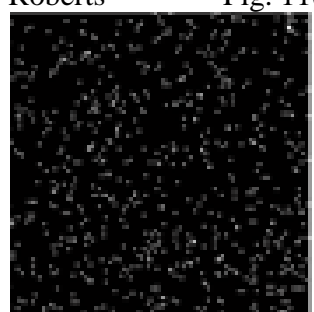

Fig. 11(e)Prewitt

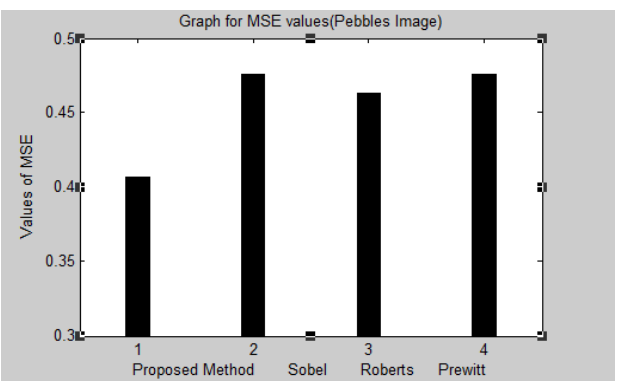

Fig. 12(a) Graph of MSE values(Pebbles Image) 


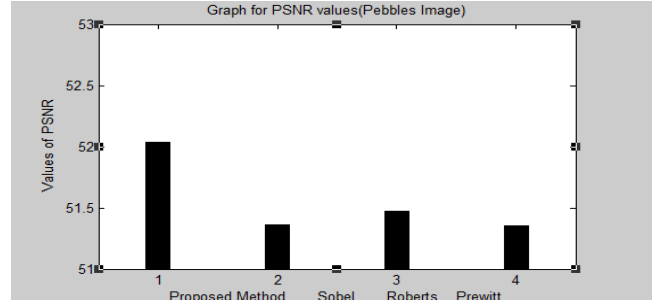

Fig. 12(b) Graph of PSNR values(Pebbles Image)

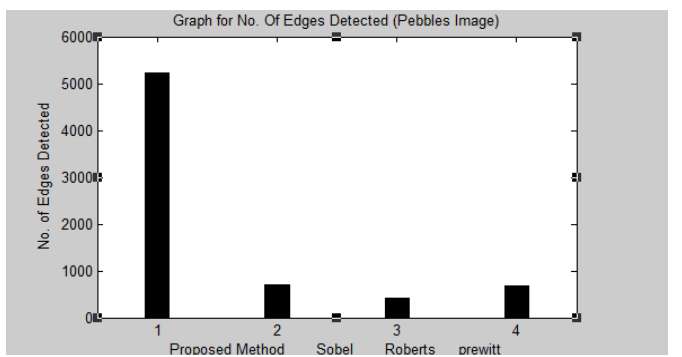

Fig. 12(c) Graph of No.of Edges detected(Pebbles Image)

Table 3: Values of MSE, PSNR and No. of Edges Detected (Pebbles Image)

\begin{tabular}{|c|c|c|c|}
\hline Methods & MSE & PSNR & $\begin{array}{c}\text { No. Of } \\
\text { Edges } \\
\text { Detected }\end{array}$ \\
\hline $\begin{array}{c}\text { Proposed } \\
\text { Method }\end{array}$ & 0.4081 & 52.0227 & 5209 \\
\hline Roberts & 0.4633 & 51.4722 & 468 \\
\hline Sobel & 0.4742 & 51.3709 & 770 \\
\hline Prewitt & 0.4750 & 51.3635 & 756 \\
& & & \\
\hline
\end{tabular}

\section{CONCLUSION}

It has been concluded that the proposed ANFIS based edge detector can be used for efficient detection of edges in digital images corrupted by salt and pepper noise. Simulation results show that the proposed ANFIS Edge detector gives outstanding results as compared to the Sobel, Roberts and Prewitt methods. Because Number of Edges detected in proposed system is more as compared to other three competing methods (Sobel, Roberts and Prewitt). The value of MSE (Mean Square Error) is lowest in the proposed method and the value of PSNR (Peak Signal to Noise Ratio) is highest for the proposed method. So it has been proved that proposed method gives better results and detects the edges more accurately. This method is suitable for various areas of digital image processing applications such as face recognition, fingerprint identification, remote sensing and medical imaging where boundaries of specific regions need to be determined for further image analysis.

\section{ACKNOWLEDGEMENT}

The authors are greatly indebted to the Department of Computer Science and Engineering, D.A.V Institute of Engineering and Technology, Jalandhar for providing excellent lab facilities that make this work possible.

\section{REFERENCES}

[1] Saket Bhardwaj, Ajay Mittal "A Survey on Various Edge Detector Techniques" Procedia Technology Elsevier ,Vol. 4,pp.220 - 226,2012.

[2] Anubhuti Khare, Manish Saxena, Shweta Tiwari "Edge Detection Method for Image Segmentation - A Survey of Soft Computing Approaches" International Journal of Soft Computing and Engineering, Vol. 1, Issue.4, pp.174-178, September 2011.

[3] M. EminYuksel and M. TulinYildirim, "A Simple Neuro-Fuzzy Edge Detector for Digital Images Corrupted by Impulse Noise", Int. J. Electron. Commun. (AEU) Elsevier, Vol.58, pp.72-75, 2004

[4] M. EminYuksel, "Edge detection in noisy images by neuro-fuzzy processing", Int. J. Electron. Commun. (AEU) Elsevier, Vol. 61, pp. 82 - 89, 2007

[5] Abdallah A. Alshennawy, And Ayman A. Aly, "Edge Detection In Digital Images Using Fuzzy Logic Technique", In proceedings of World Academy of Science Engineering and Technology, Vol.51 , pp.178186, 2009.

[6] Begol, Moslem and Maghooli,Keivan "Improving Digital Image Edge Detection by Fuzzy Systems", In proceedings of World Academy of Science, Engineering and Technology, Vol.57, pp.76-79, 2011.

[7] Lei Zhang, Mei Xiao, Jian Ma and Hongxun Song "Edge Detection by Adaptive Neuro-Fuzzy Inference System" 2nd International Conference on Image and Signal Processing,(IEEE),2009.

[8] Hamed Mehrara, Mohammad Zahedinejad and Ali Pourmohammad "Novel Edge Detection Using BP Neural Network Based on Threshold Binarization", Second International Conference on Computer and Electrical Engineering (IEEE), pp.408-412, 2009.

[9] Aborisade, D.O "Novel Fuzzy logic Based Edge Detection Technique" International Journal of Advanced Science and Technology, Vol. 29, pp.75-82, April, 2011.

[10] Shashank Mathur, Anil Ahlawat "Application Of Fuzzy Logic On Image Edge Detection" International Conference "Intelligent Information and Engineering Systems" INFOS, Varna, Bulgaria, 2008.

[11] Constantina Raluca Mihalache and Mitic a Craus "Neural Network and Fuzzy Membership Functions Based Edge Detection for Digital Images" 16th International Conference on System Theory, Control and Computing,(IEEE),2012.

[12] Jappreet Kaur, Manpreet Kaur, Poonamdeep Kaur, Manpreet Kaur "Comparative Analysis of Image Denoising Techniques" International Journal of Emerging Technology and Advanced Engineering, Vol. 2, Issue 6, pp.296-298, June 2012

[13] Mohsen Sharifi, Mahmoud Fathy, Maryam Tayefeh Mahmoudi "A Classified and Comparative Study of Edge Detection Algorithms" Proceedings of the International Conference on Information Technology: Coding and Computing, (IEEE) 2002.

[14] Om Prakash Verma ,Madasu Hanmandlu ,Ashish Kumar Sultania ,Anil Singh Parihar "A novel fuzzy system for edge detection in noisy image using bacterial foraging" Multidim Syst Sign Process Springer, Vol. 24.pp.181198,2013 